\title{
REFINEMENTS OF SOME INEQUALITIES CONCERNING THE POLAR DERIVATIVE OF A POLYNOMIAL
}

\author{
Nisar A. Rather, Suhail Gulzar
}

Abstract: If $P(z)=a_{n} z^{n}+\sum_{j=\mu}^{n} a_{n-j} z^{n-j}, 1 \leqslant \mu \leqslant n$, is a polynomial of degree $n$ having all its zeros in $|z| \leqslant k, k \leqslant 1$, then it was recently claimed by K. K. Dewan, Naresh Singh, Abdullah Mir [Extensions of some polynomial inequalities to the polar derivative, J. Math. Anal. Appl. 352 (2009), 807-815] that for every real or complex number $\alpha$, with $|\alpha| \geqslant k^{\mu}$,

$$
\begin{aligned}
\max _{|z|=1}\left|D_{\alpha} P(z)\right| \geqslant & \frac{n\left(|\alpha|-k^{\mu}\right)}{1+k^{\mu}} \max _{|z|=1}|P(z)|+\frac{n(|\alpha|+1)}{k^{n-\mu}\left(1+k^{\mu}\right)} m \\
& +n\left(\frac{k^{\mu}-A_{\mu}}{1+k^{\mu}}\right) \max _{|z|=1}|P(z)|+\frac{n\left(A_{\mu}-k^{\mu}\right)}{k^{n}\left(1+k^{\mu}\right)} m
\end{aligned}
$$

where $m=\min _{|z|=k}|P(z)|, D_{\alpha} P(z)$ is a polar derivative of $P(z)$ with respect to the point $\alpha \in \mathbb{C}$ and $A_{\mu}$ is given by (1.11). The proof of this result is not correct. In this paper, we present certain more refined results which not only provides a correct proof of above inequality as a special case but also yields a refinement of above and other related result.

Keywords: polynomials, inequalities in the complex domain, polar derivative, Bernstein's inequality.

\section{Introduction and statement of results}

If $P(z)$ is a polynomial of degree $n$, then

$$
\max _{|z|=1}\left|P^{\prime}(z)\right| \leqslant n \max _{|z|=1}|P(z)| .
$$

Inequality (1.1) is an immediate consequence of S. Bernstein's Theorem on the derivative of a trigonometric polynomial (for reference, see [13, p.531], [15, p.508] or [17]) equality in (1.1) holds for $P(z)=a z^{n}, a \neq 0$.

If we restrict ourselves to the class of polynomials of degree $n$ having no zero in $|z|<1$, then inequality $(1.1)$ can be replaced by

$$
\max _{|z|=1}\left|P^{\prime}(z)\right| \leqslant \frac{n}{2} \max _{|z|=1}|P(z)| .
$$

2010 Mathematics Subject Classification: primary: 30A10; secondary: 30C10, 30C15 
Inequality (1.2) was conjectured by Erdös and later verified by Lax [8].The result is sharp and equality holds for $P(z)=\alpha z^{n}+\beta,|\alpha|=|\beta|$.

For polynomials $P(z)$ of degree $n$ having all zeros in $|z| \leqslant 1$, it was proved by Turán [18] that

$$
\max _{|z|=1}\left|P^{\prime}(z)\right| \geqslant \frac{n}{2} \max _{|z|=1}|P(z)| .
$$

The inequality (1.3) is best possible and the extremal polynomial is $P(z)=(z+1)^{n}$.

As an extension of (1.2), Malik [12] proved that if $P(z) \neq 0$ in $|z|<k$ where $k \geqslant 1$, then

$$
\max _{|z|=1}\left|P^{\prime}(z)\right| \leqslant \frac{n}{1+k} \max _{|z|=1}|P(z)|,
$$

where as if $P(z)$ has all its zeros in $|z| \leqslant k$ where $k \leqslant 1$, then

$$
\max _{|z|=1}\left|P^{\prime}(z)\right| \geqslant \frac{n}{1+k} \max _{|z|=1}|P(z)| \text {. }
$$

By considering the class of polynomials $P(z)=a_{n} z^{n}+\sum_{j=\mu}^{n} a_{n-j} z^{n-j}$ of degree $n$ having all their zeros in $|z| \leqslant k, k \leqslant 1$, Aziz and Shah [4] proved

$$
\max _{|z|=1}\left|P^{\prime}(z)\right| \geqslant \frac{n}{1+k^{\mu}}\left\{\max _{|z|=1}|P(z)|+\frac{1}{k^{n-\mu}} \min _{|z|=k}|P(z)|\right\} .
$$

On the other hand, for the more general class of polynomials $P(z)=a_{0}+$ $\sum_{j=\mu}^{n} a_{j} z^{j}, 1 \leqslant \mu \leqslant n$, not vanishing in $|z|<k$ where $k \geqslant 1$, Gardner, Govil, Weems [9] proved

$$
\max _{|z|=1}\left|P^{\prime}(z)\right| \leqslant \frac{n}{1+s_{0}}\left\{\max _{|z|=1}|P(z)|-m\right\}
$$

where $m=\min _{|z|=k}|P(z)|$ and

$$
s_{0}=k^{\mu+1}\left\{\frac{\left(\frac{\mu}{n}\right) \frac{\left|a_{\mu}\right|}{\left|a_{0}\right|-m} k^{\mu-1}+1}{\left(\frac{\mu}{n}\right) \frac{\left|a_{\mu}\right|}{\left|a_{0}\right|-m} k^{\mu+1}+1}\right\} .
$$

In the literature (see $[2,5,9,10,11,14])$ there exist some refinements and generalizations of all the above inequalities.

Let $D_{\alpha} P(z)$ denote the polar derivative of the polynomial $P(z)$ of degree $n$ with respect to the point $\alpha \in \mathbb{C}$, then

$$
D_{\alpha} P(z)=n P(z)+(\alpha-z) P^{\prime}(z) .
$$

The polynomial $D_{\alpha} P(z)$ is of degree at most $n-1$ and it generalizes the ordinary derivative in the sense that

$$
\lim _{\alpha \rightarrow \infty} \frac{D_{\alpha} P(z)}{\alpha}=P^{\prime}(z)
$$

uniformly with respect to $z$ with $|z| \leqslant R, R>0$. 
Dewan et al. [7] (see also [16]) extended inequality (1.6) to the polar derivative and they proved that if $P(z)=a_{n} z^{n}+\sum_{j=\mu}^{n} a_{n-j} z^{n-j}, 1 \leqslant \mu \leqslant n$, is a polynomial of degree $n$ having all its zeros in $|z| \leqslant k, k \leqslant 1$, then for every complex number $\alpha$ with $|\alpha| \geqslant k^{\mu}$,

$$
\max _{|z|=1}\left|D_{\alpha} P(z)\right| \geqslant \frac{n\left(|\alpha|-k^{\mu}\right)}{1+k^{\mu}} \max _{|z|=1}|P(z)|+\frac{n(|\alpha|+1)}{k^{n-\mu}\left(1+k^{\mu}\right)} \min _{|z|=k}|P(z)| .
$$

While seeking the desired refinement of inequality (1.9), recently Dewan et al. [6] have made an incomplete attempt by claiming to have proved the following result.

Theorem 1.1. Let $P(z)=a_{n} z^{n}+\sum_{j=\mu}^{n} a_{n-j} z^{n-j}$ where $1 \leqslant \mu \leqslant n$, be a polynomial of degree $n$ having all its zeros in $|z| \leqslant k, k \leqslant 1$, then for every complex number $\alpha$ with $|\alpha| \geqslant k^{\mu}$, we have

$$
\begin{aligned}
\max _{|z|=1}\left|D_{\alpha} P(z)\right| \geqslant & \frac{n\left(|\alpha|-k^{\mu}\right)}{1+k^{\mu}} \max _{|z|=1}|P(z)|+\frac{n(|\alpha|+1)}{k^{n-\mu}\left(1+k^{\mu}\right)} m \\
& +n\left(\frac{k^{\mu}-A_{\mu}}{1+k^{\mu}}\right) \max _{|z|=1}|P(z)|+\frac{n\left(A_{\mu}-k^{\mu}\right)}{k^{n}\left(1+k^{\mu}\right)} m
\end{aligned}
$$

where $m=\min _{|z|=k}|P(z)|$ and

$$
A_{\mu}=\frac{n\left(\left|a_{n}\right|-m / k^{n}\right) k^{2 \mu}+\mu\left|a_{n-\mu}\right| k^{\mu-1}}{n\left(\left|a_{n}\right|-m / k^{n}\right) k^{\mu-1}+\mu\left|a_{n-\mu}\right|} .
$$

The proof of Theorem 1.1 given by Dewan et al. [6] is not correct. The reason being that the authors in [6] deduce in lines $8-10$ on page 814 , that for every complex number $\alpha$ with $|\alpha| \geqslant k^{\mu}, 1 \leqslant \mu \leqslant n$, the polynomial $D_{\alpha}\left[P(z)-\frac{m \lambda z^{n}}{k^{n}}\right]$ has all its zeros in $|z|<k, k \leqslant 1$ by using Lemma 7 in [6] which is not true in general for $1 \leqslant \mu \leqslant n$. Here Lemma 7 of [6] is applicable only when $\mu=1$ (see $[1,13,15]$ ). Thus the argument used to establish that all the zeros of $D_{\alpha}\left[P(z)-\frac{m \lambda z^{n}}{k^{n}}\right]$ lie in $|z|<k$ for $|\alpha| \geqslant k^{\mu}$ is false.

The immediate counterexample $P(z)=4 z^{2}-1, \mu=2$ having all its zeros in $|z|<k=3 / 5<1$ demonstrates, by taking $\alpha=2 / 5>k^{\mu}$ that the zero of $D_{\alpha} P(z)=\frac{16 z}{5}-2$ lie in $|z|>k=3 / 5$.

They [6] have also proved the following result.

Theorem 1.2. If $P(z)=a_{n} z^{n}+\sum_{j=\mu}^{n} a_{n-j} z^{n-j}, 1 \leqslant \mu \leqslant n$, is a polynomial of degree $n$ having all its zeros in $|z| \leqslant k$ where $k \leqslant 1$ and $\delta$ is any complex number with $|\delta| \leqslant 1$, then for $|z|=1$

$$
\left|D_{\delta} P(z)\right| \leqslant n\left(\frac{k^{\mu}+|\delta|}{1+k^{\mu}}\right) \max _{|z|=1}|P(z)|-\frac{n(1-|\delta|)}{k^{n-\mu}\left(1+k^{\mu}\right)} \min _{|z|=k}|P(z)| .
$$

The result is best possible and equality in (1.12) holds for $P(z)=\left(z^{\mu}+k^{\mu}\right)^{n / \mu}$, where $n$ is a multiple of $\mu$ and $\delta \geqslant 0$. The proof of Theorem 1.2 given by Dewan et. al. [6] is valid only when $P(0) \neq 0$. 
For the class of polynomials $P(z)=a_{0}+\sum_{j=\mu}^{n} a_{j} z^{j}, 1 \leqslant \mu \leqslant n$, not vanishing in $|z| \leqslant k$ where $k \geqslant 1$, N. A. Rather and M. I. Mir [16] proved the following result.

Theorem 1.3. If $P(z)=a_{0}+\sum_{j=\mu}^{n} a_{j} z^{j}, 1 \leqslant \mu \leqslant n$, is a polynomial of degree $n$ which does not vanish in $|z|<k$ where $k \geqslant 1$, then for every complex number $\beta$ with $|\beta| \leqslant k^{\mu}$,

$$
\max _{|z|=1}\left|D_{\beta} P(z)\right| \geqslant \frac{n}{1+k^{\mu}}\left\{\left(k^{\mu}-|\beta|\right) \max _{|z|=1}|P(z)|+(|\beta|+1) m\right\}
$$

where $m=\min _{|z|=k}|P(z)|$.

The main aim of this paper is to present a correct proof of Theorem 1.1 and establish some refinements of Theorems 1.1, 1.2, 1.3.

In this direction, we first present the following more general result which not only provides a correct proof of Theorem 1.1 but also yields an improvement of Theorem 1.1 and a refinement of inequality (1.6).

Theorem 1.1. Let $P(z)=a_{n} z^{n}+\sum_{j=\mu}^{n} a_{n-j} z^{n-j}, 1 \leqslant \mu \leqslant n$, be a polynomial of degree $n$ having all its zeros in $|z| \leqslant k$ where $k \leqslant 1$, then for every complex number $\alpha$ with $|\alpha| \geqslant A_{\mu}$

$$
\max _{|z|=1}\left|D_{\alpha} P(z)\right| \geqslant n\left(\frac{|\alpha|-A_{\mu}}{1+A_{\mu}}\right) \max _{|z|=1}|P(z)|+\frac{n A_{\mu}}{k^{n}}\left(\frac{1+|\alpha|}{1+A_{\mu}}\right) m
$$

where $A_{\mu}$ is given by (1.11) and $m=\min _{|z|=k}|P(z)|$.

By Lemma 2.7, $A_{\mu} \leqslant k^{\mu}$, therefore, Theorem 1.1 holds for every $\alpha$ with $|\alpha| \geqslant k^{\mu}$ as well. Also the right hand side of inequality (1.14) can be written as

$$
\begin{aligned}
& \frac{n\left(|\alpha|-k^{\mu}\right)}{\left(1+k^{\mu}\right)} \max _{|z|=1}|P(z)|+\frac{n(|\alpha|+1)}{k^{n-\mu}\left(1+k^{\mu}\right)} m \\
+ & n\left(\frac{k^{\mu}-A_{\mu}}{1+k^{\mu}}\right) \max _{|z|=1}|P(z)|+\frac{n\left(A_{\mu}-k^{\mu}\right)}{k^{n}\left(1+k^{\mu}\right)} m \\
+ & \frac{n\left(k^{\mu}-A_{\mu}\right)\left(|\alpha|-A_{\mu}\right)}{\left(1+k^{\mu}\right)\left(1+A_{\mu}\right)}\left\{\max _{|z|=1}|P(z)|-\frac{m}{k^{n}}\right\},
\end{aligned}
$$

therefore, the following interesting result which is a refinement of Theorem 1.1 follows immediately from Theorem 1.1.

Corollary 1.2. Let $P(z)=a_{n} z^{n}+\sum_{j=\mu}^{n} a_{n-j} z^{n-j}, 1 \leqslant \mu \leqslant n$, be a polynomial of degree $n$ having all its zeros in $|z| \leqslant k, k \leqslant 1$, then for every complex number $\alpha$ 
with $|\alpha| \geqslant k^{\mu}$, we have

$$
\begin{aligned}
\max _{|z|=1}\left|D_{\alpha} P(z)\right| \geqslant & \frac{n\left(|\alpha|-k^{\mu}\right)}{\left(1+k^{\mu}\right)} \max _{|z|=1}|P(z)|+\frac{n(|\alpha|+1)}{k^{n-\mu}\left(1+k^{\mu}\right)} m \\
& +n\left(\frac{k^{\mu}-A_{\mu}}{1+k^{\mu}}\right) \max _{|z|=1}|P(z)|+\frac{n\left(A_{\mu}-k^{\mu}\right)}{k^{n}\left(1+k^{\mu}\right)} m \\
& +\frac{n\left(k^{\mu}-A_{\mu}\right)\left(|\alpha|-A_{\mu}\right)}{\left(1+k^{\mu}\right)\left(1+A_{\mu}\right)}\left\{\max _{|z|=1}|P(z)|-\frac{m}{k^{n}}\right\}
\end{aligned}
$$

where $A_{\mu}$ is given by (1.11).

In fact, except the cases $k=1$ or $\frac{\mu}{n}\left(\frac{\left|a_{n-\mu}\right|}{\left|a_{n}\right|-m / k^{n}}\right)=k^{\mu}$ the bound obtained in Corollary 1.2 is always sharp than the bound obtained from Theorem 1.1 and for this it needs to show that

$$
\frac{n\left(k^{\mu}-A_{\mu}\right)\left(|\alpha|-A_{\mu}\right)}{\left(1+k^{\mu}\right)\left(1+A_{\mu}\right)}\left\{\max _{|z|=1}|P(z)|-\frac{m}{k^{n}}\right\} \geqslant 0 .
$$

In view of inequality (2.13), the inequality (1.16) becomes equivalent to

$$
\max _{|z|=1}|P(z)| \geqslant \frac{m}{k^{n}}
$$

which is true by Lemma 2.5 and hence inequality (1.16) holds.

Remark 1.3. Corollary 1.2 establishes a correct proof of a result due to Dewan et al. [6, Theorem 3] and also provides its refinement.

If we divide both sides of inequality (1.15) by $|\alpha|$ and let $|\alpha| \rightarrow \infty$, we get the following result which is a refinement of inequality (1.6).

Corollary 1.4. Let $P(z)=a_{n} z^{n}+\sum_{j=\mu}^{n} a_{n-j} z^{n-j}, 1 \leqslant \mu \leqslant n$, be a polynomial of degree $n$ having all its zeros in $|z| \leqslant k, k \leqslant 1$, then

$$
\begin{aligned}
\max _{|z|=1}\left|P^{\prime}(z)\right| \geqslant & \frac{n}{1+k^{\mu}}\left\{\max _{|z|=1}|P(z)|+\frac{1}{k^{n-\mu}} \min _{|z|=k}|P(z)|\right\} \\
& +\frac{n\left(k^{\mu}-A_{\mu}\right)}{\left(1+k^{\mu}\right)\left(1+A_{\mu}\right)}\left\{\max _{|z|=1}|P(z)|-\frac{1}{k^{n}} \min _{|z|=k}|P(z)|\right\}
\end{aligned}
$$

where $A_{\mu}$ is given by (1.11).

We next present the following result which is the refinement of theorem 1.2.

Theorem 1.5. Let $P(z)=a_{n} z^{n}+\sum_{j=\mu}^{n} a_{n-j} z^{n-j}, 1 \leqslant \mu \leqslant n, a_{0} \neq 0$, be $a$ polynomial of degree $n$ having all its zeros in $|z| \leqslant k, k \leqslant 1$, and $\delta$ is any complex number with $|\delta| \leqslant 1$, then

$$
\max _{|z|=1}\left|D_{\delta} P(z)\right| \leqslant \frac{n\left(A_{\mu}+|\delta|\right)}{1+A_{\mu}} \max _{|z|=1}|P(z)|-\frac{n A_{\mu}(1-|\delta|)}{\left(1+A_{\mu}\right) k^{n}} \min _{|z|=k}|P(z)|
$$

where $A_{\mu}$ is given by (1.11). 
It is easy to verify that Theorem 1.5 provides a refinement of Theorem 1.2 . By Lemma 2.8,

$$
n\left(\frac{x+|\delta|}{1+x}\right) \max _{|z|=1}|P(z)|-n\left(\frac{(1-|\delta|) x}{(1+x) k^{n}}\right) \min _{|z|=k}|P(z)|
$$

is non-decreasing function of $x$. Combining this fact with Lemma 2.7, according to which $A_{\mu} \leqslant k^{\mu}$ for $\mu \geqslant 1$, it follows that Theorem 1.5 is a refinement of Theorem 1.2.

As an application of Theorem 1.1, we finally present the following result which yields a refinement of Theorem 1.3.

Theorem 1.6. If $P(z)=a_{0}+\sum_{j=\mu}^{n} a_{j} z^{j}, 1 \leqslant \mu \leqslant n$, is a polynomial of degree $n$ which does not vanish in $|z|<k$ where $k \geqslant 1$, then for every complex number $\beta$ with $|\beta| \leqslant s_{0}$,

$$
\max _{|z|=1}\left|D_{\beta} P(z)\right| \geqslant \frac{n}{1+s_{0}}\left\{\left(s_{0}-|\beta|\right) \max _{|z|=1}|P(z)|+(|\beta|+1) m\right\}
$$

where $s_{0}$ is given by (1.8) and $m=\min _{|z|=k}|P(z)|$.

By Lemma 2.4, $s_{0} \geqslant k^{\mu}$. Therefore, Theorem 1.6 is also valid for $|\beta| \leqslant k^{\mu}$ and the right hand side of inequality (1.19) is equivalent to

$$
\begin{aligned}
& \frac{n}{1+k^{\mu}}\left\{\left(k^{\mu}-|\beta|\right) \max _{|z|=1}|P(z)|+(|\beta|+1) m\right\} \\
&+\frac{n\left(s_{0}-k^{\mu}\right)(1+|\beta|)}{\left(1+k^{\mu}\right)\left(1+s_{0}\right)}\left(\max _{|z|=1}|P(z)|-m\right) .
\end{aligned}
$$

Thus, in view of Lemma 2.6, Theorem 1.6 leads to the following refinement of Theorem 1.3.

Corollary 1.7. If $P(z)=a_{0}+\sum_{j=\mu}^{n} a_{j} z^{j}, 1 \leqslant \mu \leqslant n$, is a polynomial of degree $n$ which does not vanish in $|z|<k$ where $k \geqslant 1$, then for every complex number $\beta$ with $|\beta| \leqslant k^{\mu}$,

$$
\begin{aligned}
\max _{|z|=1}\left|D_{\beta} P(z)\right| \geqslant & \frac{n}{1+k^{\mu}}\left\{\left(k^{\mu}-|\beta|\right) \max _{|z|=1}|P(z)|+(|\beta|+1) m\right\} \\
& +\frac{n\left(s_{0}-k^{\mu}\right)(1+|\beta|)}{\left(1+k^{\mu}\right)\left(1+s_{0}\right)}\left(\max _{|z|=1}|P(z)|-m\right)
\end{aligned}
$$

where $s_{0}$ is given by (1.8) and $m=\min _{|z|=k}|P(z)|$. 


\section{Lemmas}

For the proof of our theorems, we need the following lemmas.

Lemma 2.1. If $P(z)=a_{n} z^{n}+\sum_{j=\mu}^{n} a_{n-j} z^{n-j}, 1 \leqslant \mu \leqslant n$, is a polynomial of degree $n$ having all its zeros in $|z| \leqslant k$ where $k \leqslant 1$ and $Q(z)=z^{n} \overline{P(1 / \bar{z})}$, then on $|z|=1$

$$
\left|Q^{\prime}(z)\right| \leqslant S_{\mu}\left|P^{\prime}(z)\right|
$$

where

$$
S_{\mu}=\frac{n\left|a_{n}\right| k^{2 \mu}+\mu\left|a_{n-\mu}\right| k^{\mu-1}}{n\left|a_{n}\right| k^{\mu-1}+\mu\left|a_{n-\mu}\right|}
$$

and

$$
\frac{\mu}{n}\left|\frac{a_{n-\mu}}{a_{n}}\right| \leqslant k^{\mu} .
$$

The above lemma is due to Aziz and Rather [3].

Lemma 2.2. If $P(z)=a_{n} z^{n}+\sum_{j=\mu}^{n} a_{n-j} z^{n-j}, 1 \leqslant \mu \leqslant n$, is a polynomial of degree $n$ having all its zeros in $|z| \leqslant k$ where $k \leqslant 1$, then for every complex $\alpha$ with $|\alpha| \geqslant S_{\mu}$,

$$
\left|D_{\alpha} P(z)\right| \geqslant n\left(\frac{|\alpha|-S_{\mu}}{1+S_{\mu}}\right)|P(z)| \quad \text { for }|z|=1 .
$$

Proof. If $Q(z)=z^{n} \overline{P(1 / \bar{z})}$, then it can be easily verified that for $|z|=1$,

$$
\begin{aligned}
\left|Q^{\prime}(z)\right| & =\left|n P(z)-z P^{\prime}(z)\right| \\
& \geqslant|n P(z)|-\left|z P^{\prime}(z)\right|,
\end{aligned}
$$

which is equivalent to

$$
\left|Q^{\prime}(z)\right|+\left|P^{\prime}(z)\right| \geqslant n|P(z)| \quad \text { for }|z|=1 .
$$

For $|z|=1$, we have by using Lemma 2.1 and inequality (2.5),

$$
\begin{aligned}
\left(1+S_{\mu}\right)\left|P^{\prime}(z)\right| & =\left|P^{\prime}(z)\right|+S_{\mu}\left|P^{\prime}(z)\right| \\
& \geqslant\left|P^{\prime}(z)\right|+\left|Q^{\prime}(z)\right| \\
& \geqslant n|P(z)|
\end{aligned}
$$

which implies,

$$
\left|P^{\prime}(z)\right| \geqslant \frac{n}{1+S_{\mu}}|P(z)| \quad \text { for }|z|=1 .
$$

Now, for every complex number $\alpha$ with $|\alpha| \geqslant S_{\mu}$,

$$
\begin{aligned}
\left|D_{\alpha} P(z)\right| & =\left|n P(z)+(\alpha-z) P^{\prime}(z)\right| \\
& \geqslant|\alpha|\left|P^{\prime}(z)\right|-\left|n P(z)-z P^{\prime}(z)\right|,
\end{aligned}
$$


which implies that for $|z|=1$,

$$
\left|D_{\alpha} P(z)\right| \geqslant|\alpha|\left|P^{\prime}(z)\right|-\left|Q^{\prime}(z)\right| .
$$

Inequality (2.7) when combined with Lemma 2.1 gives,

$$
\left|D_{\alpha} P(z)\right| \geqslant\left(|\alpha|-S_{\mu}\right)\left|P^{\prime}(z)\right| \quad \text { for }|z|=1 .
$$

The above inequality in conjunction with inequality (2.6) yields,

$$
\left|D_{\alpha} P(z)\right| \geqslant n\left(\frac{|\alpha|-S_{\mu}}{1+S_{\mu}}\right)|P(z)| .
$$

This proves Lemma 2.2.

Lemma 2.3. If $P(z)=a_{0}+\sum_{j=\mu}^{n} a_{j} z^{j}, 1 \leqslant \mu \leqslant n$, is a polynomial of degree $n$ having no zeros in $|z|<k$ where $k \geqslant 1$, then for every complex number $\alpha$ with $|\alpha| \geqslant 1$

$$
\max _{|z|=1}\left|D_{\alpha} P(z)\right| \leqslant \frac{n}{1+s_{0}}\left\{\left(|\alpha|+s_{0}\right) \max _{|z|=1}|P(z)|-(|\alpha|-1) m\right\}
$$

where $m=\min _{|z|=k}|P(z)|$ and $s_{0}$ is as defined in (1.8).

The above Lemma is due to Dewan et al. [6, Theorem 1] and the following Lemma is due to Gardner, Govil and Weems [9].

Lemma 2.4. If $P(z)=a_{0}+\sum_{j=\mu}^{n} a_{j} z^{j}, 1 \leqslant \mu \leqslant n$, is a polynomial of degree $n$ having no zeros in $|z|<k, k \geqslant 1$, then

$$
s_{0} \geqslant k^{\mu}
$$

where $s_{0}$ is given by (1.8).

Lemma 2.5. If $P(z)=\sum_{j=1}^{n} a_{j} z^{j}$ is a polynomial of degree $n$ having all its zeros in $|z| \leqslant k, k \leqslant 1$ and $m=\min _{|z|=k}|P(z)|$, then

$$
\max _{|z|=1}|P(z)| \geqslant \frac{m}{k^{n}}
$$

and, in particular,

$$
\left|a_{n}\right|>\frac{m}{k^{n}}
$$

Proof. Since the polynomial $P(z)$ has all its zeros in $|z| \leqslant k, k \leqslant 1$, the polynomial $Q(z)=z^{n} \overline{P(1 / \bar{z})}$ has no zero in $|z|<1 / k, 1 / k \geqslant 1$. We can assume without loss of generality that $Q(z)$ has no zero on $|z|=1 / k$, for otherwise the result holds trivially. Since $Q(z)$, being polynomial, is analytic for $|z| \leqslant 1 / k$ and has no zeros in $|z| \leqslant 1 / k$, by the Minimum Modulus Principle

$$
|Q(z)| \geqslant \min _{|z|=1 / k}|Q(z)| \quad \text { for }|z| \leqslant 1 / k \text { where } 1 / k \geqslant 1 .
$$


This in particular gives,

$$
|Q(z)| \geqslant \frac{1}{k^{n}} \min _{|z|=k}|P(z)| \quad \text { for } \quad|z|=1 \quad \text { and } \quad|Q(0)|>\frac{1}{k^{n}} \min _{|z|=k}|P(z)|,
$$

which implies,

$$
\max _{|z|=1}|P(z)|=\max _{|z|=1}|Q(z)| \geqslant \frac{m}{k^{n}} \quad \text { and } \quad\left|a_{n}\right|>\frac{m}{k^{n}} .
$$

This completes the proof of Lemma 2.5.

Lemma 2.6. If $P(z)=\sum_{j=1}^{n} a_{j} z^{j}$ is a polynomial of degree $n$ which does not vanish in $|z|<k$ where $k \geqslant 1$, then

$$
\max _{|z|=1}|P(z)| \geqslant \min _{|z|=k}|P(z)| \text {. }
$$

Proof. We can assume without loss of generality that $P(z)$ has no zero on $|z|=k$, for otherwise, the result holds trivially. Since $P(z)$ is analytic for $|z| \leqslant k$ and has no zeros in $|z| \leqslant k$, by the Minimum Modulus Principle

$$
|P(z)| \geqslant \min _{|z|=k}|P(z)| \quad \text { for }|z| \leqslant k \text { where } k \geqslant 1,
$$

which in particular gives,

$$
|P(z)| \geqslant \min _{|z|=k}|P(z)| \quad \text { for }|z|=1 .
$$

This proves Lemma 2.6.

Lemma 2.7. If $P(z)=a_{n} z^{n}+\sum_{j=\mu}^{n} a_{n-j} z^{n-j}, 1 \leqslant \mu \leqslant n$, is a polynomial of degree $n$ having all its zeros in $|z| \leqslant k, k \leqslant 1$, then

$$
A_{\mu} \leqslant k^{\mu}
$$

where $A_{\mu}$ is defined in (1.11).

The above result is due to Dewan et. al [6].

Lemma 2.8. The function

$$
A(x)=n\left(\frac{x+|\delta|}{1+x}\right) \max _{|z|=1}|P(z)|-n\left(\frac{(1-|\delta|) x}{(1+x) k^{n}}\right) \min _{|z|=k}|P(z)|
$$

is a non-decreasing function of $x$ for every $\delta$ with $|\delta| \leqslant 1$.

Proof. The derivative of $A(x)$ with respect to $x$ is

$$
A^{\prime}(x)=\frac{n(1-|\delta|)}{(1+x)^{2}}\left[\max _{|z|=1}|P(z)|-\frac{1}{k^{n}} \min _{|z|=k}|P(z)|\right],
$$

by Lemma 2.5 for every $\delta$ with $|\delta| \leqslant 1, A^{\prime}(x) \geqslant 0$ for all $x \neq-1$. Hence $A(x)$ is non-decreasing function of $x$. 
Lemma 2.9. The function

$$
S_{\mu}(x)=\frac{n x k^{2 \mu}+\mu\left|a_{n-\mu}\right| k^{\mu-1}}{n x k^{\mu-1}+\mu\left|a_{n-\mu}\right|},
$$

where $k \leqslant 1$ and $\mu \geqslant 1$, is a non-increasing function of $x$.

Proof. The proof follows by considering the first derivative test for $S_{\mu}(x)$.

\section{Proof of Theorems}

Proof of Theorem 1.1. By hypothesis, the polynomial $P(z)=a_{n} z^{n}+$ $\sum_{j=\mu}^{n} a_{n-j} z^{n-j}, 1 \leqslant \mu \leqslant n$, has all its zeros in $|z| \leqslant k, k \leqslant 1$. If $P(z)$ has a zero on $|z|=k$, then $m=0$ and the result follows from Lemma 2.2. Hence, we suppose that all the zeros of $P(z)$ lie in $|z|<k, k \leqslant 1$, so that $m>0$.

Now $m \leqslant|P(z)|$ for $|z|=k$, therefore, if $\lambda$ is any complex number such that $|\lambda|<1$, then

$$
\left|\frac{m \lambda z^{n}}{k^{n}}\right|<|P(z)| \quad \text { for }|z|=k .
$$

Since all the zeros of $P(z)$ lie in $|z|<k$, it follows by Rouche's theorem that all the zeros of

$$
F(z)=P(z)-\frac{m \lambda z^{n}}{k^{n}}=\left(a_{n}-\frac{\lambda m}{k^{n}}\right) z^{n}+\sum_{j=\mu}^{n} a_{n-j} z^{n-j}
$$

also lie in $|z|<k, k \leqslant 1$. Applying Lemma 2.1 to the polynomial $F(z)$, we get for $|z|=1$,

$$
S_{\mu}^{\prime}\left|F^{\prime}(z)\right| \geqslant\left|G^{\prime}(z)\right|
$$

where $G(z)=z^{n} \overline{F(1 / \bar{z})}=z^{n} \overline{P(1 / \bar{z})}+\frac{m \bar{\lambda}}{k^{n}}$ and

$$
S_{\mu}^{\prime}=\frac{n\left|a_{n}-\frac{m \lambda}{k^{n}}\right| k^{2 \mu}+\mu\left|a_{n-\mu}\right| k^{\mu-1}}{n\left|a_{n}-\frac{m \lambda}{k^{n}}\right| k^{\mu-1}+\mu\left|a_{n-\mu}\right|} .
$$

Since by Lemma 2.5, $\left|a_{n}\right|>\frac{m}{k^{n}}$, therefore, for every $\lambda$ with $|\lambda|<1$, we have

$$
\left|a_{n}-\frac{m \lambda}{k^{n}}\right| \geqslant\left|a_{n}\right|-\frac{m|\lambda|}{k^{n}} \geqslant\left|a_{n}\right|-\frac{m}{k^{n}} .
$$

Now combining (3.2), (3.3) and Lemma 2.9 for every $\lambda$ with $|\lambda|<1$, we get

$$
\begin{aligned}
S_{\mu}^{\prime} & =\frac{n\left|a_{n}-\frac{m \lambda}{k^{n}}\right| k^{2 \mu}+\mu\left|a_{n-\mu}\right| k^{\mu-1}}{n\left|a_{n}-\frac{m \lambda}{k^{n}}\right| k^{\mu-1}+\mu\left|a_{n-\mu}\right|} \\
& \leqslant \frac{n\left(\left|a_{n}\right|-\frac{m}{k^{n}}\right) k^{2 \mu}+\mu\left|a_{n-\mu}\right| k^{\mu-1}}{n\left(\left|a_{n}\right|-\frac{m}{k^{n}}\right) k^{\mu-1}+\mu\left|a_{n-\mu}\right|}=A_{\mu} \quad \text { (say). }
\end{aligned}
$$


Using inequality (3.4) in inequality (3.1), we obtain

$$
A_{\mu}\left|F^{\prime}(z)\right| \geqslant\left|G^{\prime}(z)\right| \quad \text { for }|z|=1 .
$$

Equivalently for $|z|=1$, we have

$$
A_{\mu}\left|P^{\prime}(z)-\frac{\lambda m n z^{n-1}}{k^{n}}\right| \geqslant\left|Q^{\prime}(z)\right|
$$

where $Q(z)=z^{n} \overline{P(1 / \bar{z})}$. Since all the zeros of polynomial $F(z)=P(z)-\frac{m \lambda z^{n}}{k^{n}}$ lie in $|z|<k$, where $k \leqslant 1$, it follows by Gauss-Lucas Theorem that all the zeros of the polynomial $T(z)=P^{\prime}(z)-\frac{\lambda m n z^{n-1}}{k^{n}}$ also lie in $|z|<k, k \leqslant 1$ for every $\lambda$ with $|\lambda|<1$. This implies

$$
\left|P^{\prime}(z)\right| \geqslant \frac{m n|z|^{n-1}}{k^{n}} \quad \text { for }|z| \geqslant k .
$$

If inequality (3.7) is not true, then there exists a point $z_{0}$ with $\left|z_{0}\right| \geqslant k$ such that

$$
\left|P^{\prime}\left(z_{0}\right)\right|<\frac{m n\left|z_{0}\right|^{n-1}}{k^{n}} .
$$

We take $\lambda=k^{n} P^{\prime}\left(z_{0}\right) / m n z_{0}^{n-1}$, then $|\lambda|<1$ and with this choice of $\lambda$ we get $T\left(z_{0}\right)=0,\left|z_{0}\right| \geqslant k$ which is clearly a contradiction to the fact that all the zeros of $T(z)$ lie in $|z|<k$. Thus inequality (3.7) holds.

Now choosing the argument of $\lambda$ in the left hand side of inequality (3.6) such that

$$
A_{\mu}\left|P^{\prime}(z)-\frac{\lambda m n z^{n-1}}{k^{n}}\right|=A_{\mu}\left\{\left|P^{\prime}(z)\right|-\frac{|\lambda| m n|z|^{n-1}}{k^{n}}\right\} \quad \text { for }|z|=1,
$$

which is possible by (3.7), we get

$$
A_{\mu}\left|P^{\prime}(z)\right|-A_{\mu} \frac{|\lambda| m n|z|^{n-1}}{k^{n}} \geqslant\left|Q^{\prime}(z)\right| \quad \text { for }|z|=1 .
$$

Letting $|\lambda| \rightarrow 1$, we obtain

$$
A_{\mu}\left|P^{\prime}(z)\right|-A_{\mu} \frac{m n}{k^{n}} \geqslant\left|Q^{\prime}(z)\right| \quad \text { for }|z|=1 .
$$

Since $Q(z)=z^{n} \overline{P(1 / \bar{z})}$, it can be easily seen that

$$
\left|Q^{\prime}(z)\right|=\left|n P(z)-z P^{\prime}(z)\right| \quad \text { for }|z|=1 .
$$

This gives for every $\alpha$ with $|\alpha| \geqslant A_{\mu}$ and for $|z|=1$,

$$
\begin{aligned}
\left|D_{\alpha} P(z)\right| & =\left|n P(z)+(\alpha-z) P^{\prime}(z)\right| \\
& \geqslant|\alpha|\left|P^{\prime}(z)\right|-\left|n P(z)-z P^{\prime}(z)\right| \\
& =|\alpha|\left|P^{\prime}(z)\right|-\left|Q^{\prime}(z)\right| .
\end{aligned}
$$


Combining inequality (3.10) with inequality (3.9), we get for $|z|=1$,

$$
\left|D_{\alpha} P(z)\right| \geqslant\left(|\alpha|-A_{\mu}\right)\left|P^{\prime}(z)\right|+A_{\mu} \frac{m n}{k^{n}} .
$$

Also, from (3.10), we have

$$
A_{\mu}\left|D_{\alpha} P(z)\right| \geqslant|\alpha| A_{\mu}\left|P^{\prime}(z)\right|-A_{\mu}\left|Q^{\prime}(z)\right| \text { for }|z|=1,
$$

which gives with the help of (3.9) for $|z|=1$ and $|\alpha| \geqslant A_{\mu}$,

$$
\begin{aligned}
A_{\mu}\left|D_{\alpha} P(z)\right| & \geqslant|\alpha|\left\{\left|Q^{\prime}(z)\right|+A_{\mu} \frac{m n}{k^{n}}\right\}-A_{\mu}\left|Q^{\prime}(z)\right| \\
& =\left(|\alpha|-A_{\mu}\right)\left|Q^{\prime}(z)\right|+A_{\mu}|\alpha| \frac{m n}{k^{n}} .
\end{aligned}
$$

Adding (3.11) and (3.12), we obtain for every complex number $\alpha$ with $|\alpha| \geqslant A_{\mu}$ and for $|z|=1$,

$$
\begin{aligned}
\left(1+A_{\mu}\right)\left|D_{\alpha} P(z)\right| & \geqslant\left(|\alpha|-A_{\mu}\right)\left\{\left|P^{\prime}(z)\right|+\left|Q^{\prime}(z)\right|\right\}+A_{\mu} \frac{m n(|\alpha|+1)}{k^{n}} \\
& =\left(|\alpha|-A_{\mu}\right)\left\{\left|z P^{\prime}(z)\right|+\left|n P(z)-z P^{\prime}(z)\right|\right\}+A_{\mu} \frac{m n(|\alpha|+1)}{k^{n}} \\
& \geqslant\left(|\alpha|-A_{\mu}\right)\left\{\left|z P^{\prime}(z)+n P(z)-z P^{\prime}(z)\right|\right\}+A_{\mu} \frac{m n(|\alpha|+1)}{k^{n}} \\
& =n\left(|\alpha|-A_{\mu}\right)|P(z)|+A_{\mu} \frac{m n(|\alpha|+1)}{k^{n}}
\end{aligned}
$$

which implies,

$$
\max _{|z|=1}\left|D_{\alpha} P(z)\right| \geqslant n\left(\frac{|\alpha|-A_{\mu}}{1+A_{\mu}}\right) \max _{|z|=1}|P(z)|+\frac{n}{k^{n}}\left(\frac{(1+|\alpha|) A_{\mu}}{1+A_{\mu}}\right) m .
$$

This completes the proof of Theorem 1.1.

Proof of Theorem 1.5. By hypothesis the polynomial $P(z)=a_{n} z^{n}+$ $\sum_{j=\mu}^{n} a_{n-\mu} z^{n-\mu}, 1 \leqslant \mu \leqslant n$, has all its zeros in $|z| \leqslant k, k \leqslant 1$, therefore the polynomial $Q(z)=z^{n} \overline{P(1 / \bar{z})}$ has no zero in $|z|<1 / k, 1 / k \geqslant 1$. Applying Lemma 2.3 to $Q(z)$, we get for every complex $\alpha$ with $|\alpha| \geqslant 1$,

$$
\left|D_{\alpha} Q(z)\right| \leqslant \frac{n}{1+s_{0}^{\prime}}\left\{\left(|\alpha|+s_{0}^{\prime}\right) \max _{|z|=1}|Q(z)|-(|\alpha|-1) \min _{|z|=1 / k}|Q(z)|\right\},
$$

where

$$
\begin{aligned}
s_{0}^{\prime} & =\frac{1}{k^{\mu+1}}\left\{\frac{\frac{\mu}{n}\left(\frac{\left|a_{n-\mu}\right|}{\left|a_{n}\right|-\min _{|z|=1 / k}|Q(z)|}\right) \frac{1}{k^{\mu-1}}+1}{\frac{\mu}{n}\left(\frac{\left|a_{n-\mu}\right|}{\left|a_{n}\right|-\min _{|z|=1 / k}|Q(z)|}\right) \frac{1}{k^{\mu+1}}+1}\right\} \\
= & \frac{\mu\left|a_{n-\mu}\right|+n\left(\left|a_{n}\right|-\frac{m}{k^{n}}\right) k^{\mu-1}}{\mu\left|a_{n-\mu}\right| k^{\mu-1}+n\left(\left|a_{n}\right|-\frac{m}{k^{n}}\right) k^{2 \mu}}=\frac{1}{A_{\mu}} .
\end{aligned}
$$


Using (3.14) in (3.13), we obtain for $|\alpha| \geqslant 1$ and $|z|=1$

$$
\begin{aligned}
\left|D_{\alpha} Q(z)\right| & \leqslant \frac{n}{1+\frac{1}{A_{\mu}}}\left\{\left(|\alpha|+\frac{1}{A_{\mu}}\right) \max _{|z|=1}|P(z)|-\frac{|\alpha|-1}{k^{n}} \min _{|z|=k}|P(z)|\right\} \\
& =\frac{n\left(|\alpha| A_{\mu}+1\right)}{1+A_{\mu}} \max _{|z|=1}|P(z)|-\frac{n A_{\mu}(|\alpha|-1)}{\left(1+A_{\mu}\right) k^{n}} \min _{|z|=k}|P(z)| .
\end{aligned}
$$

If $|z|=1$ so that $z \bar{z}=1$, then we have

$$
\begin{aligned}
\left|D_{\alpha} Q(z)\right| & =\left|n Q(z)+(\alpha-z) Q^{\prime}(z)\right| \\
& =\left|n z^{n} \overline{P(1 / \bar{z})}+(\alpha-z)\left\{n z^{n-1} \overline{P(1 / \bar{z})}-z^{n-2} \overline{P^{\prime}(1 / \bar{z})}\right\}\right| \\
& =\left|\alpha\left\{n z^{n-1} \overline{P(1 / \bar{z})}-z^{n-2} \overline{P^{\prime}(1 / \bar{z})}\right\}+z^{n-1} \overline{P^{\prime}(1 / \bar{z})}\right| \\
& =\left|\alpha\left(n \overline{P(z)}-\bar{z} \overline{P^{\prime}(z)}\right)+\overline{P^{\prime}(z)}\right| \\
& =\left|\bar{\alpha} n P(z)+(1-\bar{\alpha} z) P^{\prime}(z)\right|=|\bar{\alpha}|\left|D_{1 / \bar{\alpha}} P(z)\right| .
\end{aligned}
$$

This gives,

$$
\left|D_{\alpha} Q(z)\right|=|\alpha|\left|D_{1 / \bar{\alpha}} P(z)\right| \quad \text { for }|\alpha| \geqslant 1 \quad \text { and } \quad|z|=1
$$

Inequality (3.16) in conjunction with (3.15) implies for $|\alpha| \geqslant 1$ and $|z|=1$,

$$
|\alpha|\left|D_{1 / \bar{\alpha}} P(z)\right| \leqslant \frac{n\left(|\alpha| A_{\mu}+1\right)}{1+A_{\mu}} \max _{|z|=1}|P(z)|-\frac{n A_{\mu}(|\alpha|-1)}{\left(1+A_{\mu}\right) k^{n}} \min _{|z|=k}|P(z)| .
$$

Replacing $1 / \bar{\alpha}$ by $\delta$, we obtain for $|\delta| \leqslant 1$ and $|z|=1$,

$$
\left|D_{\delta} P(z)\right| \leqslant \frac{n\left(A_{\mu}+|\delta|\right)}{1+A_{\mu}} \max _{|z|=1}|P(z)|-\frac{n A_{\mu}(1-|\delta|)}{\left(1+A_{\mu}\right) k^{n}} \min _{|z|=k}|P(z)|,
$$

which proves Theorem 1.5.

Proof of Theorem 1.6. Since all the zeros of polynomial $P(z)=a_{0}+\sum_{j=\mu}^{n} a_{j} z^{j}$, $1 \leqslant \mu \leqslant n$, lie in $|z| \geqslant k$, where $k \geqslant 1$, all the zeros of polynomial $Q(z)=$ $z^{n} \overline{P(1 / \bar{z})}=\overline{a_{0}} z^{n}+\sum_{j=\mu}^{n} \overline{a_{j}} z^{n-j}, 1 \leqslant \mu \leqslant n$ lie in $|z| \leqslant 1 / k \leqslant 1$. Applying Theorem 1.1 to the polynomial $Q(z)$ and noting that $\min _{|z|=1 / k}|Q(z)|=$ $1 / k^{n} \min _{|z|=k}|P(z)|$, we get for $|\alpha| \geqslant A_{\mu}^{\prime}$,

$$
\max _{|z|=1}\left|D_{\alpha} Q(z)\right| \geqslant n\left(\frac{|\alpha|-A_{\mu}^{\prime}}{1+A_{\mu}^{\prime}}\right) \max _{|z|=1}|Q(z)|+n k^{n}\left(\frac{(1+|\alpha|) A_{\mu}^{\prime}}{1+A_{\mu}^{\prime}}\right) \min _{|z|=1 / k}|Q(z)|
$$


where

$$
\begin{aligned}
A_{\mu}^{\prime} & =\frac{n\left(\left|a_{0}\right|-k^{n} \min _{|z|=1 / k}|Q(z)|\right) \frac{1}{k^{2 \mu}}+\mu\left|a_{\mu}\right| \frac{1}{k^{\mu-1}}}{n\left(\left|a_{0}\right|-k^{n} \min _{|z|=1 / k}|Q(z)|\right) \frac{1}{k^{\mu-1}}+\mu\left|a_{\mu}\right|} \\
= & \frac{1}{k^{\mu+1}}\left\{\frac{\left(\frac{\mu}{n}\right) \frac{\left|a_{\mu}\right|}{\left|a_{0}\right|-m} k^{\mu+1}+1}{\left(\frac{\mu}{n}\right) \frac{\left|a_{\mu}\right|}{\left|a_{0}\right|-m} k^{\mu-1}+1}\right\}=\frac{1}{s_{0}} .
\end{aligned}
$$

Equivalently,

$$
\max _{|z|=1}\left|D_{\alpha} Q(z)\right| \geqslant n\left(\frac{|\alpha| s_{0}-1}{1+s_{0}}\right) \max _{|z|=1}|P(z)|+n\left(\frac{1+|\alpha|}{1+s_{0}}\right) \min _{|z|=k}|P(z)| .
$$

Using (3.16) in (3.18), we get

$$
\max _{|z|=1}\left(|\bar{\alpha}|\left|D_{1 / \bar{\alpha}} P(z)\right|\right) \geqslant n\left(\frac{|\alpha| s_{0}-1}{1+s_{0}}\right) \max _{|z|=1}|P(z)|+n\left(\frac{1+|\alpha|}{1+s_{0}}\right) \min _{|z|=k}|P(z)| .
$$

Setting $\beta=1 / \bar{\alpha}$ so that $|\beta| \leqslant s_{0}$, we obtain

$$
\max _{|z|=1}\left|D_{\beta} P(z)\right| \geqslant n\left(\frac{s_{0}-|\beta|}{1+s_{0}}\right) \max _{|z|=1}|P(z)|+n\left(\frac{|\beta|+1}{1+s_{0}}\right) \min _{|z|=k}|P(z)| .
$$

This proves Theorem 1.6.

Acknowledgement. The authors are highly grateful to the referee for his valuable suggestions and comments. The second author is supported by Council of Scientific and Industrial Research, New Delhi, under grant F.No. 09/251(0047)/2012EMR-I.

\section{References}

[1] A. Aziz, A new proof of Laguerre's theorem about the zeros of polynomials, Bull. Austral. Math. Soc. 33 (1986), 131-138.

[2] A. Aziz and Q.M. Dawood, Inequalities for a polynomial and its derivatives, J. Approx. Theory 54 (1998), 306-311.

[3] A. Aziz and N.A. Rather, Some Zygmund type $L^{q}$ inequalities for polynomials, J. Math. Anal. Appl. 289 (2004), 14-29.

[4] A. Aziz and W.M. Shah, An integral mean estimate for polynomial, Indian J. Pure Appl. Math. 28 (1997), 1413-1419.

[5] T.N. Chan and M.A. Malik, On Erdös-Lax theorem, Proc. Indian Acad. Sci. 92 (1983), 191-193.

[6] K.K. Dewan, N. Singh and A. Mir, Extensions of some polynomial inequalities to the polar derivative, J. Math. Anal. Appl. 352 (2009), 807-815. 
[7] K.K. Dewan, N. Singh and R. Lal, Inequalities for the polar derivatives of polynomials, Int. J. Pure Appl. Math. 33 (2006), 109-117.

[8] P.D. Lax, Proof of a conjecture of P. Erdös on the derivative of a polynomial, Bull. Amer. Math. Soc. 50 (1944), 509-513

[9] R.B. Gardner, N.K. Govil and A. Weems, Some results concerning rate of growth of polynomials, East J. Approx. 10 (2004), 301-312.

[10] N.K. Govil, Some inequalities for derivatives of polynomials, J. Approx. Theory 66 (1991), no. 1, 29-35.

[11] N.K. Govil, Q.I. Rahman and G. Schmeisser, On the derivative of a polynomial, Illinois J. Math. 23 (1979), 319-329.

[12] M.A. Malik, On the derivative of a polynomial, J. Lond. Math. Soc., Second Series 1 (1969), 57-60.

[13] G.V. Milovanovic, D.S. Mitrinovic and Th. M. Rassias, Topics in Polynomials: Extremal Properties, Inequalities, Zeros, World scientific Publishing Co., Singapore, (1994).

[14] M.A. Qazi, On the maximum modulus of polynomials, Proc. Amer. Math. Soc. 115 (1992), 337-343.

[15] Q.I. Rahman and G. Schmessier, Analytic theory of polynomials, Claredon Press, Oxford, 2002.

[16] N.A. Rather and M.I. Mir, Some refinements of inequalities for the polar derivative of polynomials with restricted zeros, Int. J. Pure and Appl. Math. 41 (2007), 1065-1074.

[17] A.C. Schaffer, Inequalities of A. Markoff and S. Bernstein for polynomials and related functions, Bull. Amer. Math. Soc. 47 (1941), 565-579.

[18] P. Turán, Uüber die Ableitung von Polynomen, Compositio Mathematica 7 (1939), 89-95.

Address: Nisar A. Rather, Suhail Gulzar: Department of Mathematics, University of Kashmir, Srinagar, Hazratbal 190006, India.

E-mail: dr.narather@gmail.com,sgmattoo@gmail.com

Received: 22 April 2013; revised: 26 February 2014 